\title{
CORRESPONDENCE
}

\section{Prognostic indices for COPD patient management: how many do we need?}

\section{To the Editors:}

Prognostic indices represent clinicians' and scientists' best intentions to improve the care of patients. Implicit in the development of indices is that they will allow a better understanding of patients' needs in relation to an underlying disease. Chronic obstructive pulmonary disease (COPD) is a chronic respiratory condition with a high impact on patients' wellbeing, health care utilisation and mortality [1]. To date, 15 prognostic indices for COPD patients have been reported since the publication of the BODE (body mass index, obstruction, dyspnoea and exercise) index in 2004 [2-14]. All indices except one were developed or enhanced for clinical use. However, in current COPD guidelines, airflow obstruction is still the only structured measure that guides clinicians on treatment decisions, along with information on patients' subjective wellbeing at the time of their visit.

ESTEBAN et al. [15] developed a potentially useful prognostic instrument for COPD patients to predict their mortality: COPD classification and analysis tree (CART). Their validation method was fairly good due to the use of a separate validation cohort, analysing both COPD-CART's discriminative potency and accuracy, and comparing COPD-CART with some previously reported indices (BODE, HADO (health, activity, dyspnoea and obstruction) and ADO (age, dyspnoea and obstruction)) [16]. However, in order to value its added predictive quality, we wonder how this new index compares to the classic standard measurement, i.e. obstruction, in their validation cohort.

Above all, it is essential to justify the utilisation of indices before recommending that practitioners use them. Since COPD-CART was developed for clinical utilisation and claims to be of better use than existing indices, our most important question does not concern its validity but whether this instrument will indeed affect patient care. The British Medical Journal has previously published a series on prognosis studies, with an emphasis on the need for impact studies that demonstrate improvement of decision making and patient outcome when using an index to guide patient management [17]. Despite various validation methods of the still growing number of COPD prognostic indices, none of these indices has revealed its impact in daily practice yet. The proof of the pudding is in the eating: good intentions could turn out to have less impact than anticipated, or may even do more harm than good.

W.D. van Dijk, T.R.J. Schermer and C. van Weel Dept of Primary and Community Care, Radboud University Nijmegen Medical Centre, Nijmegen, The Netherlands.
Correspondence: W.D. van Dijk, Dept of Primary and Community Care, Radboud University Nijmegen Medical Centre, Internal postal code 117, PO Box 9101, Nijmegen, 6500HB, The Netherlands. E-mail: w.vandijk@aios.umcn.nl

Support Statement: The PhD project of W.D. van Dijk was supported by a university grant (RvB08.066.51196/GE) from Radboud University Nijmegen Medical Centre (Nijmegen, the Netherlands).

Statement of Interest: Statements of interest for T.R.J. Schermer and C. van Weel can be found at www.erj.ersjournals.com/site/ misc/statements.xhtml

\section{REFERENCES}

1 Global Initiative for Chronic Obstructive Lung Disease. Global Strategy for the Diagnosis, Management and Prevention of COPD (updated 2010). www.goldcopd.org/uploads/users/files/GOLD Report_April112011.pdf.

2 Puhan MA, Garcia-Aymerich J, Frey M, et al. Expansion of the prognostic assessment of patients with chronic obstructive pulmonary disease: the updated BODE index and the ADO index. Lancet 2009; 374: 704-711.

3 Celli BR, Cote CG, Marin JM, et al. The body-mass index, airflow obstruction, dyspnoea, and exercise capacity index in chronic obstructive pulmonary disease. N Engl J Med 2004; 350: 1005-1012.

4 Esteban C, Quintana JM, Aburto M, et al. A simple score for assessing stable chronic obstructive pulmonary disease. QJM 2006; 99: 751-759.

5 Briggs A, Spencer M, Wang H, et al. Development and validation of a prognostic index for health outcomes in chronic obstructive pulmonary disease. Arch Intern Med 2008; 168: 71-79.

6 Kostianev SS, Hodgev VA, Iluchev DH. Multidimensional system for assessment of COPD patients. Comparison with BODE index. Folia Med (Plovdiv) 2008; 50: 29-38.

7 Mehrotra N, Freire AX, Bauer DC, et al. Predictors of mortality in elderly subjects with obstructive airway disease: the PILE score. Ann Epidemiol 2010; 20: 223-232.

8 Soler-Cataluna JJ, Martinez-Garcia MA, Sanchez LS, et al. Severe exacerbations and BODE index: two independent risk factors for death in male COPD patients. Respir Med 2009; 103: 692-699.

9 Omachi TA, Yelin EH, Katz PP, et al. The COPD severity score: a dynamic prediction tool for health-care utilization. COPD 2008; 5: 339-346.

10 Jones RC, Donaldson GC, Chavannes NH, et al. derivation and validation of a composite index of severity in chronic obstructive pulmonary disease - the DOSE Index. Am J Respir Crit Care Med 2009; 180: 1189-1195.

11 Niewoehner DE, Lokhnygina $\mathrm{Y}$, Rice $\mathrm{K}$, et al. Risk indexes for exacerbations and hospitalizations due to COPD. Chest 2007; 131: $20-28$. 
12 Azarisman MS, Fauzi MA, Faizal MP, et al. The SAFE (SGRQ score, air-flow limitation and exercise tolerance) Index: a new composite score for the stratification of severity in chronic obstructive pulmonary disease. Postgrad Med J 2007; 83: 492-497.

13 Cardoso F, Tufanin AT, Colucci M, et al. Replacement of the 6-min walk test with maximal oxygen consumption in the BODE Index applied to patients with COPD: an equivalency study. Chest 2007; 132: 477-482.

14 Schembri S, Anderson W, Morant S, et al. A predictive model of hospitalisation and death from chronic obstructive pulmonary disease. Respir Med 2009; 103: 1461-1467.
15 Esteban C, Arostegui I, Moraza J, et al. Development of a decision tree to assess the severity and prognosis of stable COPD. Eur Respir J 2011; 38: 1294-1300.

16 Altman DG, Vergouwe $Y$, Royston $\mathrm{P}$, et al. Prognosis and prognostic research: validating a prognostic model. BMJ 2009; 338: b605.

17 Moons KG, Altman DG, Vergouwe Y, et al. Prognosis and prognostic research: application and impact of prognostic models in clinical practice. BMJ 2009; 338: b606.

DOI: 10.1183/09031936.00088011

\section{Avoiding backward steps in COPD: looking again at}

\section{roflumilast}

\section{To the Editors:}

I read with interest the article by BATEMAN et al. [1] on the influence of roflumilast when it is used concomitantly with longacting $\beta_{2}$-agonists to reduce exacerbations. Similarly, roflumilast remained effective with concomitant short-acting muscarinic antagonists. Phosphodiesterase (PDE) 4 inhibitors have arisen as anti-inflammatory drugs that may prevent exacerbations in moderate to severe chronic obstructive pulmonary disease (COPD) patients who fulfil the chronic bronchitis profile. Preclinical investigations suggest that roflumilast mitigates tobacco smoke-induced lung inflammation, mucociliary malfunction, lung fibrotic remodelling, oxidative stress or pulmonary vascular remodelling. However, clinical studies have failed to identify inflammation biomarkers that demonstrate significant and evident anti-inflammatory effects of roflumilast.

There are two potential controversies from the basic research. First, COPD may be understood from the point of view of PDE4 dysfunction, which may explain systemic COPD manifestations through mechanisms alternative to those solely of inflammation. PDE4 has been implicated in fetal survival and growth, control of airway smooth muscle tone, adaptation velocity of contraction in cardiac myocytes, mood regulation, neutrophil recruitment, and promotion of the tumour necrosis factor (TNF)- $\alpha$ inflammation pathway. Knockout models and pharmacological inhibition of PDE4 have also demonstrated antidepressant effects and triggers for arrhythmias or heart failure. All these findings have led to the study of the role of PDE4 in heart failure, cardiovascular risk, sarcopenia, osteoporosis, anaemia and depression. Interestingly, these are associated comorbidities currently described as extrapulmonary manifestations of COPD [2]. Conversely, PDE4 mutations and polymorphisms, initially described in neuropsychiatric disorders or ischaemic stroke, have also been recently encountered in COPD or osteopenia. Homma et al. [3] investigated the association between the polymorphisms of the PDE4D gene and the development of COPD. They found significant differences between the COPD and control groups in the distribution of SNP13 allele frequencies of the PDE4D gene and haplotypes composed of PDE4D SNP7 and IL13 +2044 G/A. This may suggest that a group of COPD patients corresponds to PDE4 altered phenotypes whose systemic manifestations may improve in response to roflumilast.
Secondly, roflumilast may act not as an anti-inflammatory drug but as a bronchodilator with additional systemic effects through PDE inhibition. When the clinical trials were analysed, compared with all other treatments, the combination of roflumilast plus long-acting muscarinic antagonist exhibited the largest treatment effects [4]. This is predictable, taking into account this inhibition of airway hyperreactivity to muscarinic cholinergic agonists. Cyclic nucleotides play a critical role in the control of smooth muscle relaxation by intersecting the $\mathrm{Ca}^{2+}$-signalling pathway that promotes contraction at several critical points. When a mouse model of allergic asthma was compared with wild-type littermates, PDE4D knockout mice sensitised intraperitoneally and challenged intranasally with ovoalbumin do not exhibit airway hyperreactivity to the muscarinic cholinergic agonist methacholine [5]. The effect of PDE4 ablation appears to be specific for muscarinic cholinergic stimulation because it is possible to induce contraction by other constrictors, such as serotonin or vasopressin. In fact, the primary end-point of the tiotropium plus roflumilast trial (M2-128 study) was change in forced expiratory volume in $1 \mathrm{~s}$, which was demonstrated.

In conclusion, it would be a serious backward step from the previous advances on COPD and PDE inhibitors if new and on-going investigations only focus on airways, and do not consider clinical evidence of inflammation. None of the clinical trials are long enough to test results on inflammation processes. Likewise, they do not investigate inflammation and compare biomarkers between roflumilast and placebo groups. It is uncertain whether roflumilast can stop inflammation in the mild COPD patient in the long term, or whether it will become inefficient after a 1-yr treatment in the moderate-tosevere patient. I hypothesise that the group of COPD with systemic manifestations may have PDE4 dysfunction and the response to PDE inhibitors could be clinically and biochemically superior to the other COPD groups. I suggest further investigations with biomarkers, such as those of endothelial dysfunction (P-selectin, intercellular adhesion molecule (ICAM)-1, ICAM-3, vascular cell adhesion molecule-1 and Eselectin) and adipokines (apolipoprotein A1, apolipoprotein B, lipoprotein A and paraoxonase), which are closely associated with the inflammatory process observed in patients with other inflammatory chronic diseases that follow the TNF- $\alpha$ pathway. 